\title{
Top sheath flow-assisted secondary flow particle manipulation in microchannels with the slanted groove structure
}

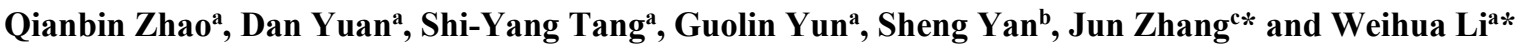 \\ a School of Mechanical, Materials, Mechatronic and Biomedical Engineering, University of Wollongong, \\ Wollongong, NSW 2522, Australia, Fax: +61 24221 3238; Tel: +61 24221 3490; E-mail: weihuali@uow.edu.au \\ ${ }^{\mathrm{b}}$ Department of Chemistry, University of Tokyo, Tokyo, Japan. \\ c Queensland Micro and Nanotechnology Centre, Griffith University, Brisbane, QLD 4111, Australia, \\ jun.zhang@griffith.edu.au
}

\begin{abstract}
In the regime of inertial microfluidics, the secondary flow is widely adopted to reduce the number of the equilibrium position and improve the focusing performance of particles. At the same time, secondary flow can also enhance the mixing effect and may deteriorate particle focusing due to the induced rotating streams, especially for the particle with a small size. In a double-layered microchannel with slanted groove structures, it has been demonstrated that the generated secondary flow at a high flow rate could focus the particle with a large size $(>8$ $\mu m)$. However, the manipulation of small size particles $(<8 \mu \mathrm{m})$ was unsuccessful as the effects of secondary flow on the small size particles was not strong enough. In this work, in order to manipulate the small size particle, we proposed a scheme to utilize a top sheath flow to enhance the focusing efficiency of the structure-induced secondary flow. The effects of the total flow rate and the flow rate ratio between the sheath and sample flow were investigated comprehensively in a large range. The $4.8 \mu \mathrm{m}$ particle could be manipulated effectively at different flow rates with the assistance of appropriate sheath flows. Besides, the effects of other factors, such as the quantity of the expansion groove structure, particle concentration and size, on particle focusing performance were also investigated. We found that the particles with the diameter of $2.9 \mu \mathrm{m}$ can also be effectively focused within the double-layered microchannel. Furthermore, we demonstrated the continuous plasma extraction from the undiluted whole blood using this proposed technique to validate its potential biological applications. The results show that the purity of plasma extracted could reach up to $\sim 99 \%$ after a single process. As such, this demonstrated sheath flow-assisted particle manipulating method can overcome the limitations of conventional design and offer much better performance for controlling smaller particles. Such a platform may enable great potential in the applications of biological and diagnostic assays, for bioparticles smaller than the normal cell size.
\end{abstract}




\section{Introduction}

Inertial microfluidics, which relies on the finite inertia and viscosity of the fluid, is one of the most popular passive microfluidic technologies for the particle manipulation in the last few decades [1, 2]. Inertial microfluidics normally works within a middle Reynolds number ranging from $\sim 1$ to $\sim 100$ [3]. As a result, it always has an inherent high throughput compared with the active microfluidic technologies, which rely on the external force fields for functionalities [3, 4].

Inertial migration is the phenomenon that randomly travelling particles can be guided to several equilibrium positions within a long enough straight channel $[5,6]$. The mechanism of this phenomenon is generally recognised as the counteraction between two intriguing inertial effects, the shear gradient lift force $F_{\mathrm{s}}$ and the wall lift force $F_{\mathrm{w}}$ [4]. At the same time, it has been reported that the equilibrium position in the straight channel is closely related with the channel aspect ratio [7-10]. Moreover, the dimension of the channel's cross-section is required to satisfy the condition, $a / D_{\mathrm{h}}>0.07$ so that the inertial migration could be strong enough to produce the obvious phenomenon [1], here $D_{\mathrm{h}}$ is the hydraulic diameter of channel, and $a$ is the diameter of particle. To manipulate particles more effectively and efficiently, the secondary flow can be introduced by the curved channel or the obstacle structures within the channel's cross sections for the generated proper pressure gradient $[11,12]$.

The double-layered microchannel with expansion groove structure was first employed for the mixing application by the secondary flow in microfluidics $[13,14]$. Then it has been demonstrated that this type of channel can also be used to regulate and improve the original inertial particle focusing performance at a high Reynolds number [9]. In our previous work, a microchannel with arc-shaped groove arrays was proposed to focus and separate particles using the groove structure-induced secondary flow. This method could focus the particles whose size is larger than $8 \mu \mathrm{m}$. However the smaller-sized particles $(4.8 \mu \mathrm{m}$ and $3.2 \mu \mathrm{m})$ were distributed relatively evenly within the microchannel, and even a number of small particles would be trapped into the expansion groove structure due to the weak resistance against the trapping effect of the pressure gradient in the expansion groove structure [15]. In the similar double-layered microchannel, a DEP-active hydrophoretic method was introduced to focus the smaller size particle [16]. For the typical hydrophoretic particle focusing method, the experimental data showed that there is a target particle dimension limitation that only the particle with a diameter larger than the half of the channel's height is able to be manipulated effectively by the effects of anisotropic expansion structure of the microchannel [17]. The integrated electrodes patterned on the microchannel bottom would generate a nonuniform electric field. Then, small size particles experienced a negative DEP force and were repelled from the channel bottom to a narrow space close to the expansion structure. As a result, the particles were prone to be focused and aligned due to the effects of the hydrophoretic steric hindrance [16]. However, this hybrid method which took the advantage of the passive microfluidic technology-hydrophoresis, and the active microfluidic technology-DEP, had the inherent problems as well. A complex set of external equipment was needed to generate and regulate the electrical field and the throughput was still much lower because particles need to be exposed to the DEP force for a sufficient amount of time to achieve successful focusing $[18,19]$.

In this work, we proposed an innovative microparticle manipulating method to overcome the limitations of the previously reported approaches for controlling smaller microparticles $(<8 \mu \mathrm{m})$ using the channel with the slanted groove structure with the introduction of a sheath flow. A single sheath flow was introduced into the double-layer microchannel from the microchannel top wall, and the suspended particles could be pushed to the bottom area of the microchannel. In this area, the structure-induced secondary flow was much more stable along 
with a single direction and the stirring effect was very weak so that even the smaller-sized particle (4.8, 3.2 and $2.9 \mu \mathrm{m}$ ) could be focused effectively. We investigated the effects of the total flow rate and the flow rate ratio between sheath flow and sample flow on the manipulation performance. In addition, other factors including the quantity of the expansion groove structure, particle concentration and size on particle focusing performance were also studied to evaluate the manipulation performance of this method. Finally, we successfully demonstrated the blood plasma extraction from undiluted whole blood using this method. It was shown that the blood plasma of $99 \%$ purity could be achieved after a single process and the result proves the robust performance of this method on small biological cell manipulation.

\section{Working Mechanism}

The double-layered microchannel with the expansion slanted groove structure was fabricated by the two-step photolithography technology shown as the Fig. 1(a) [12]. The first layer is a straight channel with a rectangular cross-section of $200 \mu m \times 26 \mu m$ (width $(W) \times$ height $(H)$ ). For the second layer, the arrays are composed of 100 slanted groove structures arranged with $38 \mu \mathrm{m}$ spacing $\left(D_{\mathrm{s}}\right)$ and oblique angle of $10^{\circ}$ on the top surface of the first layer-channel. The height $\left(H_{\mathrm{g}}\right)$ and width $\left(W_{\mathrm{g}}\right)$ of the expansion structure are 24 and $40 \mu \mathrm{m}$, respectively.

To investigate the mechanism of this sheath flow-enhanced secondary flow manipulation method, COMSOL Multiphysics 5.1 (Burlington, MA) was used to simulate the flow field within a segment of the channel with characteristic expansion groove structure. A 3D model was built and imported into COMSOL. The steady laminar flow physics was selected for the model because of the low Reynolds number of channel in microfluidics. The velocity of flow was set as $2.9162 \mathrm{~m} / \mathrm{s}$ at the inlet. The boundary condition was set as no slip and the component was meshed by the free tetrahedral mesh at the default finer level to ensure the accuracy of simulation results. In Fig. 1(b), it is obvious that an ellipse-shaped secondary flow was generated within the cross sections of the microchannel because of the pressure gradient induced by the expansion groove structure. The cross sections were divided into several areas according to the characteristic of flow order to help to explain the manipulation theory clearly. The grey rectangular area, which was close to the interface between the straight channel and expansion groove structure, was regarded as the "trapping area". In this region, it could be found that the secondary flow tended to drive the randomly distributed particles into the expansion groove structure as the direction of the secondary flow biased to the expansion structure. This bias could drive the particles into the expansion groove structure if the size of the particle was not large enough. When the sheath flow was introduced from the top surface of the channel, the particles, which were initially distributed randomly within the microchannel, could be pushed downward and restricted within the bottom "working area" of the microchannel (dark blue rectangular area). In this area, the direction of the secondary flow was mainly pointing toward the horizontal direction constantly. As a result, the particles were moving horizontally in one direction and finally arrived at the "focusing area" (green rectangular area) on the left side of the microchannel. However, we also observed that the magnitude of the secondary flow in the "working area" and "focusing area" was relatively weak compared with other areas within the cross sections because of the "no-slip" boundary condition. And this might be the reason why the particles were not able to be focused into a single tight streamline in the experiments, but rather as a particle distribution band instead.

\section{Results and discussion}

Effects of the total flow rate and flow rate ratio 
In this work, the particles with the diameter of 4.8 (CAT. NO. G0500, 5\%CV), 3.2 (CAT. NO. R0300, 5\%CV) and $2.9 \mu \mathrm{m}$ (CAT. NO. G0300, 5\%CV) were suspended in the deionized (DI) water respectively, with the concentration of $5 \times 10^{4}$ counts $/ \mathrm{ml}$. The surfactant, Tween 20 (Sigma-Aldrich Product no. P9416), was added at the ratio of $0.1 \% \mathrm{w} / \mathrm{v}$ in the particle suspensions to prevent the particles from aggregation and adhesion to the walls of the microchannel.

We conducted a series of experiments of $4.8 \mu \mathrm{m}$ particles at different flow conditions to investigate the effects of the flow rate and flow rate ratio between the sheath flow and sample flow. The total flow rate was increased from 200 to $700 \mu \mathrm{l} / \mathrm{min}$. In general, a higher total flow rate and flow rate ratio of sheath flow to sample flow can improve the particle focusing performance of this double-layered channel. Based on the captured fluorescent patterns of the $4.8 \mu \mathrm{m}$ particle (Fig. 2(a)), at the flow rate of 200 to $300 \mu \mathrm{l} / \mathrm{min}$, particles were not able to be focused effectively with all the tested flow rate ratios, and the phenomenon that $4.8 \mu \mathrm{m}$ particles were trapped and travelling spirally within the expansion groove structure could be observed. However, there was still a clear trend that the trapping phenomenon was weakened and a number of particles were gradually focused to one side of the channel as the flow rate and flow rate ratio increased. When the flow rate was increased to $400 \mu \mathrm{l} / \mathrm{min}$, the focusing performance of $4.8 \mu \mathrm{m}$ particles was further improved due to the generation of a stronger secondary flow. In addition, a higher flow rate ratio was also found to be able to enhance the focusing performance, as the stronger sheath flow could further push and restrict the particles into a narrower band within the "working area", which benefited the particle manipulation performance. When the flow rate was between 500 to $700 \mu \mathrm{l} / \mathrm{min}$, we found that particles could be guided to one side of the microchannel to be focused as a band with all the tested ratios (from 1 to 6). And the focusing performance was also slightly enhanced that the focused particle band became relatively narrower at such a higher flow rate ratio shown as the particle distribution plots in Fig. 2(b).

\section{Other contributing factors}

Besides the flow rate and flow rate ratio, we also studied the effects of the quantity of the expansion groove structure, as well as the particle concentration and size on the manipulating performance. First, the progress of the $4.8 \mu \mathrm{m}$ particle focusing along different quantities of groove structures at two different flow conditions were investigated, as shown in Fig. 3(a). When the flow rate was at $400 \mu \mathrm{l} / \mathrm{min}$ and flow rate ratio at 1, only a part of the particles could be guided to the equilibrium position and the remaining particles were still randomly distributed and the phenomenon of particle trapping in the grooves can be observed obviously. On the contrary, at the flow rate of $600 \mu \mathrm{l} / \mathrm{min}$ and the flow rate ratio of 2 , all particles could be manipulated effectively and guided towards the equilibrium position at the $75^{\text {th }}$ segment of the channel and no trapping phenomenon was observed throughout the whole process. Next, we examined the focusing performance using a particle suspension with a higher concentration of the particle $\left(2.5 \times 10^{5}\right.$ counts $\left./ \mathrm{ml}\right)$ using the same microchannel at the same flow condition, as shown in Fig. 3(b), from which we could see that the particles could be focused into a narrow band similar to the case of low particle concentration. Finally, at the flow rate of $700 \mu \mathrm{l} / \mathrm{min}$ and the flow rate ratio of 5, smaller-sized particles (3.2 and $2.9 \mu \mathrm{m}$ ) were observed to be focused in a narrow band similar as the $4.8 \mu \mathrm{m}$ particle (Fig. 3(c)).

\section{Blood plasma extraction from the whole blood}

It is known that the plasma is a straw-coloured liquid component, which contains a diversity of biological analytes, such as the proteins, metabolites, exosomes and circulating nucleic acids (CNAs) [20]. Besides the blood plasma, nearly $45 \%$ of the whole blood volume is made up of the different blood cells including the erythrocytes, leukocytes and platelets. Therefore, the plasma extraction is a necessary process to eliminate the interference of 
blood cells for an accurate and reliable detection result [21]. Although extensive works on the extraction of blood plasma using microfluidic devices have been reported, extraction of blood plasma from undiluted blood is a great challenge due to the high concentration of blood cells [22, 23]. And some plasma extraction methods using microfluidic technology has been summarized in Table 1. To prove the practical functionality of this microfluidic manipulating method, we conducted blood plasma extraction from the human whole blood (45\% het) using the proposed platform.

In our experiments, the sample of whole blood that was collected from a healthy donor using vacutainer tubes (Vacuette) containing anticoagulant agent lithium heparin was injected into the microchannel at the flow rate of $100 \mu \mathrm{l} / \mathrm{min}$, meanwhile a sheath flow of phosphate-buffered saline (PBS) (Sigma-Aldrich Product no. P5493-1L) at $400 \mu \mathrm{l} / \mathrm{min}$ was applied. From the bright field images given in Fig. 4(a)i, most blood cells were pushed towards one side of the microchannel, however, there were still a large number of blood cells trapped into the expansion groove structure. Blood cells were travelling within the expansion groove structure and then get back to the straight channel in the opposite direction. As a result, many blood cells were still distributed randomly and not able to be guided to the focusing area, which would influence and decrease the purity of the plasma at the outlet. Using the same microchannel at the same flow condition (total flow rate of $500 \mu \mathrm{l} / \mathrm{min}$ and the flow rate ratio of 4 ), the blood cells could be focused effectively when the whole blood sample was diluted by $\times 8$ times $(5.63 \%$ hct), and nearly pure plasma could be obtained from the outlet Fig. 4(a)ii.

To further enhance the performance of plasma extraction for the undiluted whole blood, the total flow rate was increased to $700 \mu \mathrm{l} / \mathrm{min}$ and the flow rate ratio was increased to $6(100 \mu \mathrm{l} / \mathrm{min}$ of the sample flow and 600 $\mu \mathrm{l} / \mathrm{min}$ of the sheath flow), it could be found that all blood cells in the undiluted blood were generally pushed towards the focusing area by the structure-induced secondary flow, as shown in Fig. 4(a)iii. Afterwards, we put the blood sample before and after extraction in a haemocytometer (Qiu Jian Biochemical Reagents Instrument co.), and the bright field images showed that countless blood cells were stacked up layer by layer from the whole blood sample at the inlet. On the contrary, only very few blood cells could be found in the extracted plasma at the outlet, Fig. 4(b). Meanwhile, both blood samples were diluted 20 times by PBS and further tested by the flow cytometry, as shown in Fig. 6(c). There were 258850 events detected in the whole blood plasma at the inlet and only 222 events detected in the extracted plasma at the outlet. This result validated that a blood plasma with purity over $99.9 \%$ could be extracted from the whole blood after a single process.

\section{Conclusion}

In conclusions, we employed a single sheath flow in the microchannel with the expansion slanted groove structure. It was proven that the suspended small particles can be gathered within the area near the bottom of the microchannel. As a result, the stable secondary flow induced by the pressure gradient would guide the small particles towards one side of the channel, and the high pressure of the assisting sheath flow can prevent the trapping of small size particles into the expansion groove structure. Finally, we demonstrated that blood plasma with a high purity of over $99.9 \%$ can be extracted from the undiluted human whole blood sample at a total flow rate of $700 \mu \mathrm{l} / \mathrm{min}$ and flow rate ratio of 6 . These characteristics enable our method to be able to serve as a great tool for separation or filtration of cells, as well as easy integration with the downstream complex detection.

\section{Acknowledgement}

J. Zhang greatly acknowledges the support from the National Natural Science Foundation of China (Grant No. 51705257), the Natural Science Foundation of Jiangsu Province (Grant No. BK20170839) and the Griffith 
University-Peking University Collaborative Travel Grant. W. Li acknowledges the support from Australian Research Council Discovery Project (Grant No. 180100055). This work is partially supported by UOW-CSC Scholarship and Dr Sheng Yan is the recipient of JSPS postdoctoral fellowship.

\section{Reference}

1. Di Carlo, D., et al., Continuous inertial focusing, ordering, and separation of particles in microchannels. Proceedings of the National Academy of Sciences, 2007. 104(48): p. 18892-18897.

2. Warkiani, M.E., et al., Slanted spiral microfluidics for the ultra-fast, label-free isolation of circulating tumor cells. Lab on a Chip, 2014. 14(1): p. 128-137.

3. Di Carlo, D., Inertial microfluidics. Lab on a Chip, 2009. 9(21): p. 3038-3046.

4. Zhang, J., et al., Fundamentals and applications of inertial microfluidics: a review. Lab on a Chip, 2016. 16(1): p. 10-34.

5. Segre, G. and A. Silberberg, Radial particle displacements in Poiseuille flow of suspensions. Nature, 1961. 189(4760): p. 209.

6. Segre, G. and A. Silberberg, Behaviour of macroscopic rigid spheres in Poiseuille flow Part 2. Experimental results and interpretation. Journal of fluid mechanics, 1962. 14(1): p. 136-157.

7. Choi, Y.-S., K.-W. Seo, and S.-J. Lee, Lateral and cross-lateral focusing of spherical particles in a square microchannel. Lab on a Chip, 2011. 11(3): p. 460-465.

8. Lee, M.G., S. Choi, and J.-K. Park, Inertial separation in a contraction-expansion array microchannel. Journal of Chromatography A, 2011. 1218(27): p. 4138-4143.

9. Chung, A.J., D.R. Gossett, and D. Di Carlo, Three dimensional, sheathless, and high-throughput microparticle inertial focusing through geometry-induced secondary flows. Small, 2013. 9(5): p. 685690 .

10. Zhou, J. and I. Papautsky, Fundamentals of inertial focusing in microchannels. Lab on a Chip, 2013. 13(6): p. 1121-1132.

11. Berger, S., L. Talbot, and L. Yao, Flow in curved pipes. Annual review of fluid mechanics, 1983. 15(1): p. 461-512.

12. Zhao, Q., et al., High-throughput sheathless and three-dimensional microparticle focusing using a microchannel with arc-shaped groove arrays. Scientific reports, 2017. 7: p. 41153.

13. Stroock, A.D., et al., Chaotic mixer for microchannels. Science, 2002. 295(5555): p. 647-651.

14. Lynn, N.S. and D.S. Dandy, Geometrical optimization of helical flow in grooved micromixers. Lab on a Chip, 2007. 7(5): p. 580-587.

15. Zhao, Q., et al., Flow rate-insensitive microparticle separation and filtration using a microchannel with arc-shaped groove arrays. Microfluidics and Nanofluidics, 2017. 21(3): p. 55.

16. Yan, S., et al., Isolating plasma from blood using a dielectrophoresis-active hydrophoretic device. Lab on a Chip, 2014. 14(16): p. 2993-3003.

17. Choi, S., et al., Hydrophoretic high-throughput selection of platelets in physiological shear-stress range. Lab on a Chip, 2011. 11(3): p. 413-418.

18. Nakashima, Y., S. Hata, and T. Yasuda, Blood plasma separation and extraction from a minute amount of blood using dielectrophoretic and capillary forces. Sensors and Actuators B: Chemical, 2010. 145(1): p. 561-569.

19. Çetin, B. and D. Li, Dielectrophoresis in microfluidics technology. Electrophoresis, 2011. 32(18): p. 2410-2427.

20. Kersaudy-Kerhoas, M. and E. Sollier, Micro-scale blood plasma separation: from acoustophoresis to egg-beaters. Lab on a Chip, 2013. 13(17): p. 3323-3346.

21. Davis, J.A., et al., Deterministic hydrodynamics: taking blood apart. Proceedings of the National Academy of Sciences, 2006. 103(40): p. 14779-14784.

22. Gossett, D.R., et al., Label-free cell separation and sorting in microfluidic systems. Analytical and bioanalytical chemistry, 2010. 397(8): p. 3249-3267.

23. Hou, H.W., et al., Microfluidic devices for blood fractionation. Micromachines, 2011. 2(3): p. $319-343$.

24. Xiang, N. and Z. Ni, High-throughput blood cell focusing and plasma isolation using spiral inertial microfluidic devices. Biomedical microdevices, 2015. 17(6): p. 110.

25. Xiang, N., et al., Inertial microfluidic syringe cell concentrator. Analytical chemistry, 2018. 90(15): p. 9515-9522.

26. Zhang, J., et al., High throughput extraction of plasma using a secondary flow-aided inertial microfluidic device. RSC Advances, 2014. 4(63): p. 33149-33159.

27. Haeberle, S., et al., Centrifugal extraction of plasma from whole blood on a rotating disk. Lab on a Chip, 2006. 6(6): p. 776-781. 
28. Lenshof, A., et al., Acoustic whole blood plasmapheresis chip for prostate specific antigen microarray diagnostics. Analytical chemistry, 2009. 81(15): p. 6030-6037.

(a)

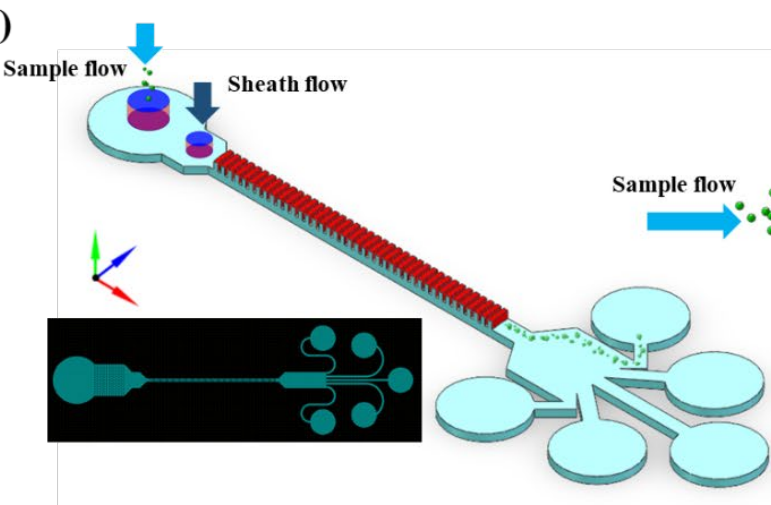

(b)

Sheath flow

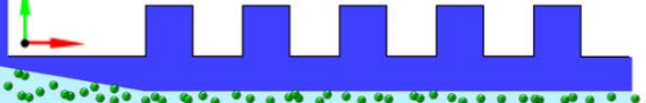

(c)

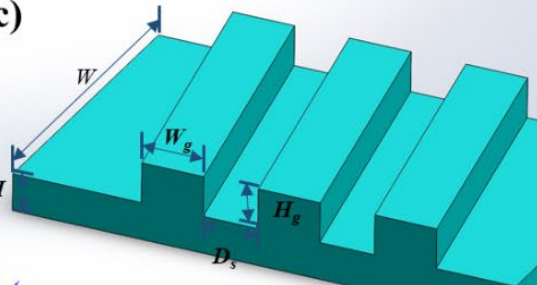

K
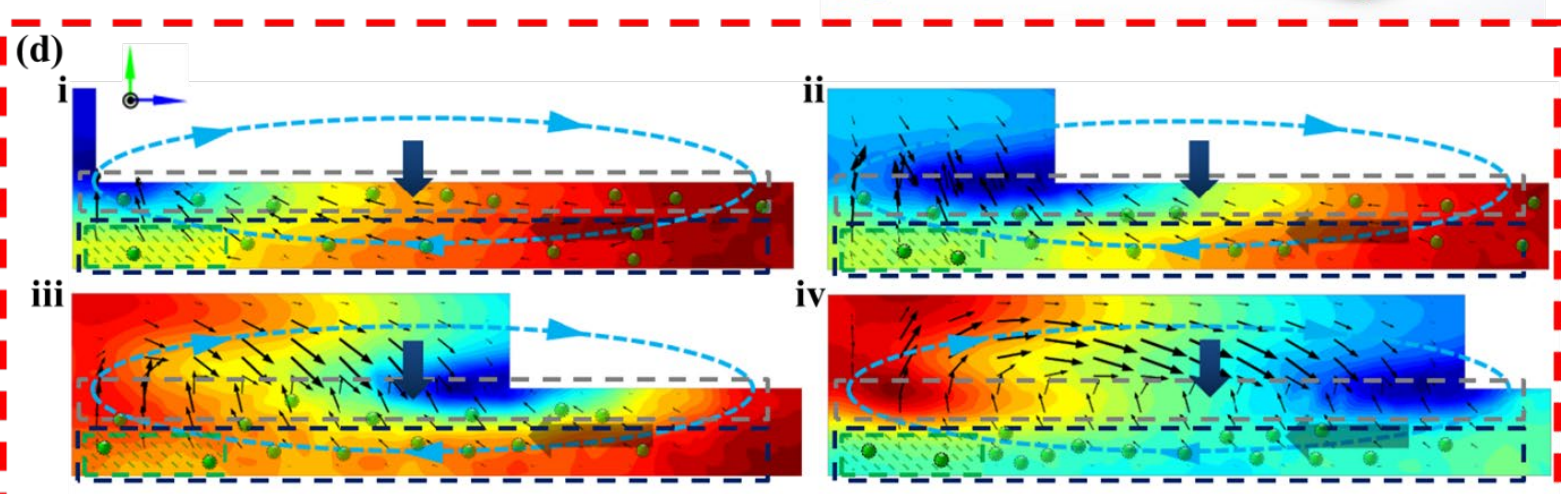

iv

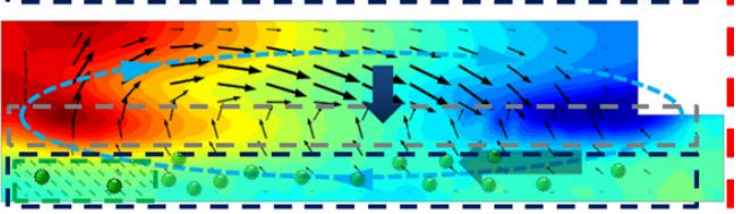

I v

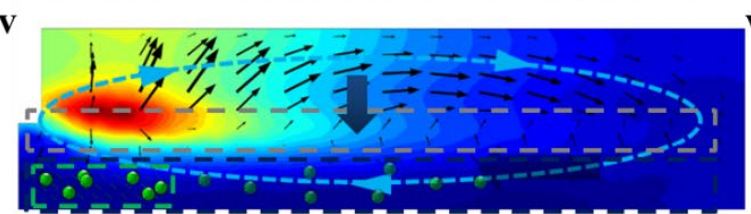

vi

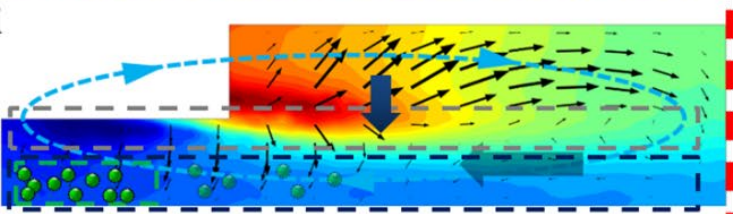

vii
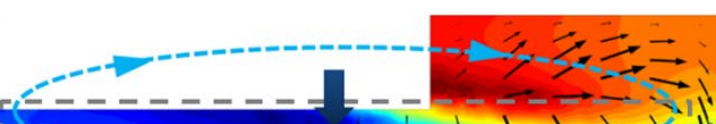

viii

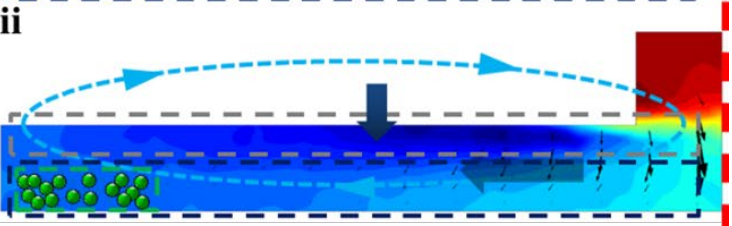

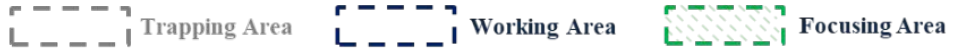

Induced

Fig. 1(a)The schematic drawing and the pattern of the microchannel used in the experiment. (b)The side view of the microchannel. (c)Dimensions of the characteristic expansion slanted groove structure of the channel. (d)Finite element analysis of the flow field within a segment of the microchannel with the characteristic expansion slanted groove structure using COMSOL Multiphysics. The 3D model was divided into 8 cross sections (i-viii) to illustrate the disturbed flow field. Each image was composed of the velocity arrow plot and pressure contour plot together. 
(a)

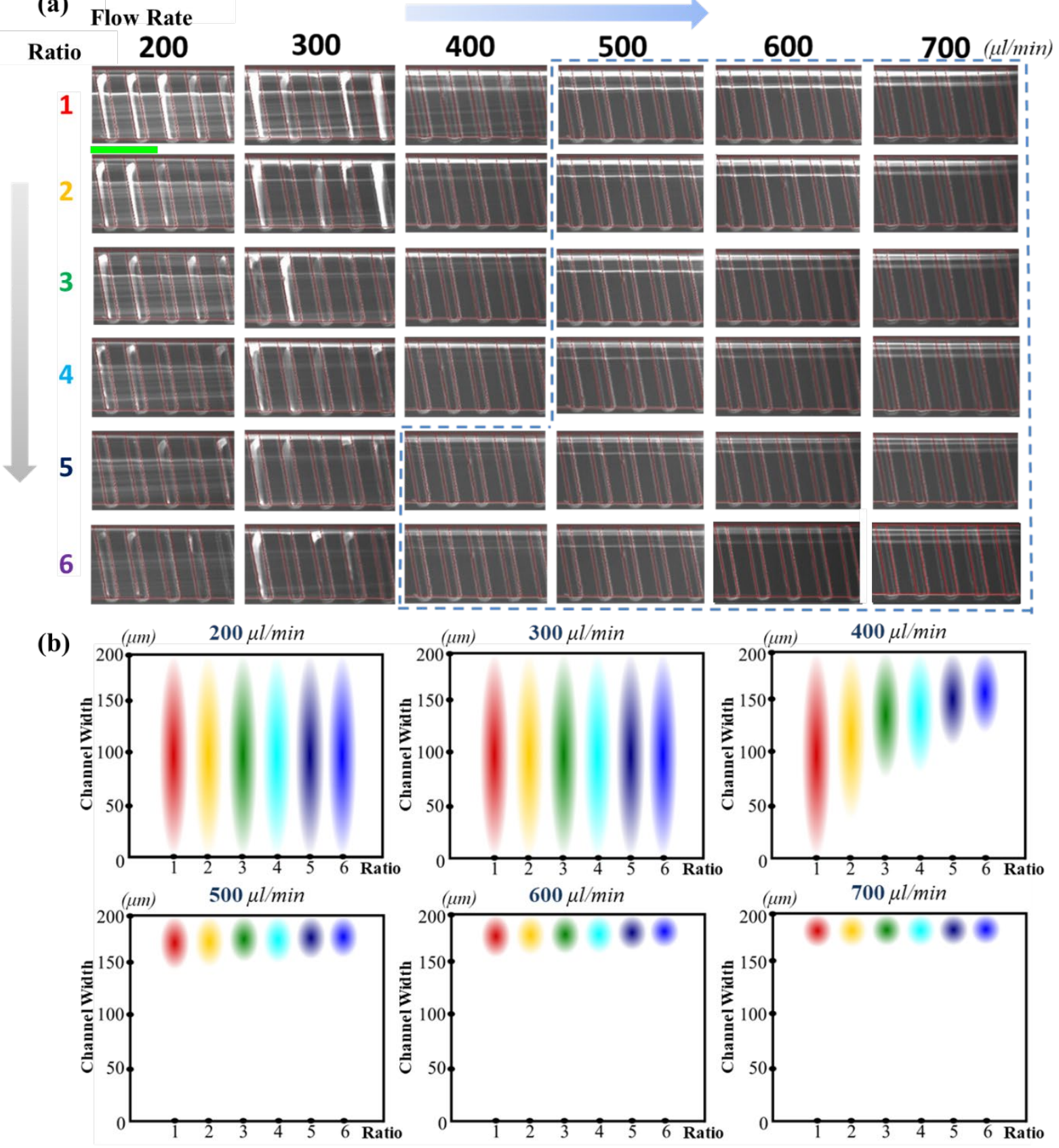

Fig. 2 (a) Fluorescent microscope images of the $4.8 \mu \mathrm{m}$ particle trajectory pattern at different flow conditions. The flow rate (the sum of the sheath flow rate and sample flow rate) was increased from $200 \mu \mathrm{l} / \mathrm{min}$ to $700 \mu \mathrm{l} / \mathrm{min}$ and the flow rate ratio of sheath flow to sample flow was ranging from 1 to 6 . The scale bar is $200 \mu \mathrm{m}$. (b)Distribution plots of $4.8 \mu \mathrm{m}$ particles at the flow rate from $200 \mu \mathrm{l} / \mathrm{min}$ to $700 \mu \mathrm{l} / \mathrm{min}$ and different ratios between the sheath flow and sample flow. All these plots were depicted according to the achieved fluorescent intensity data of particle trajectory pattern. 

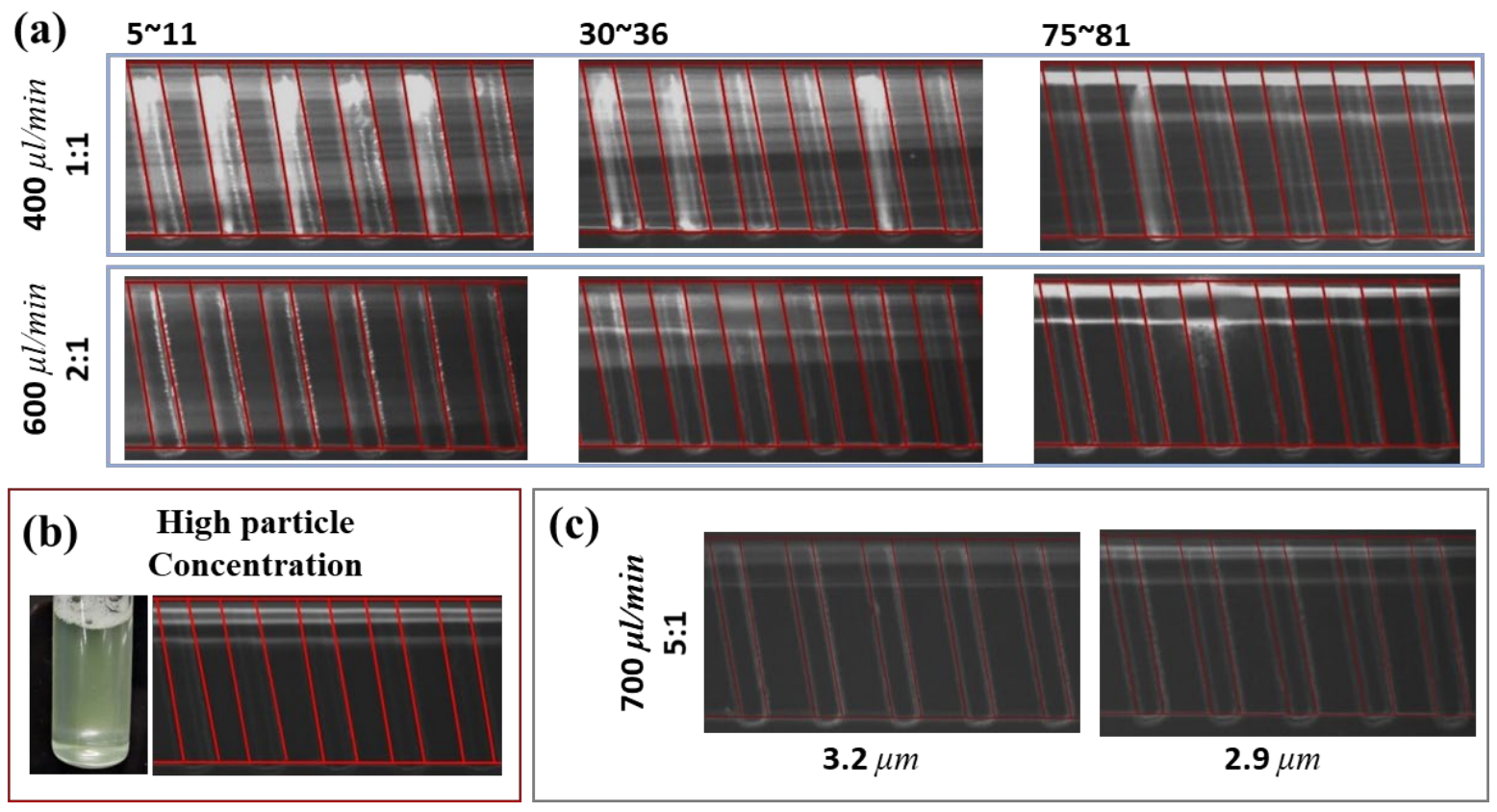

Fig. 3 (a) $4.8 \mu \mathrm{m}$ particles migration progress with different segments in the microchannel at two flow conditions. (b)The particle manipulation for a high concentration particle suspension. (c)The manipulation performance of the particle of $3.2 \mu \mathrm{m}$ and $2.9 \mu \mathrm{m}$. 
(a)
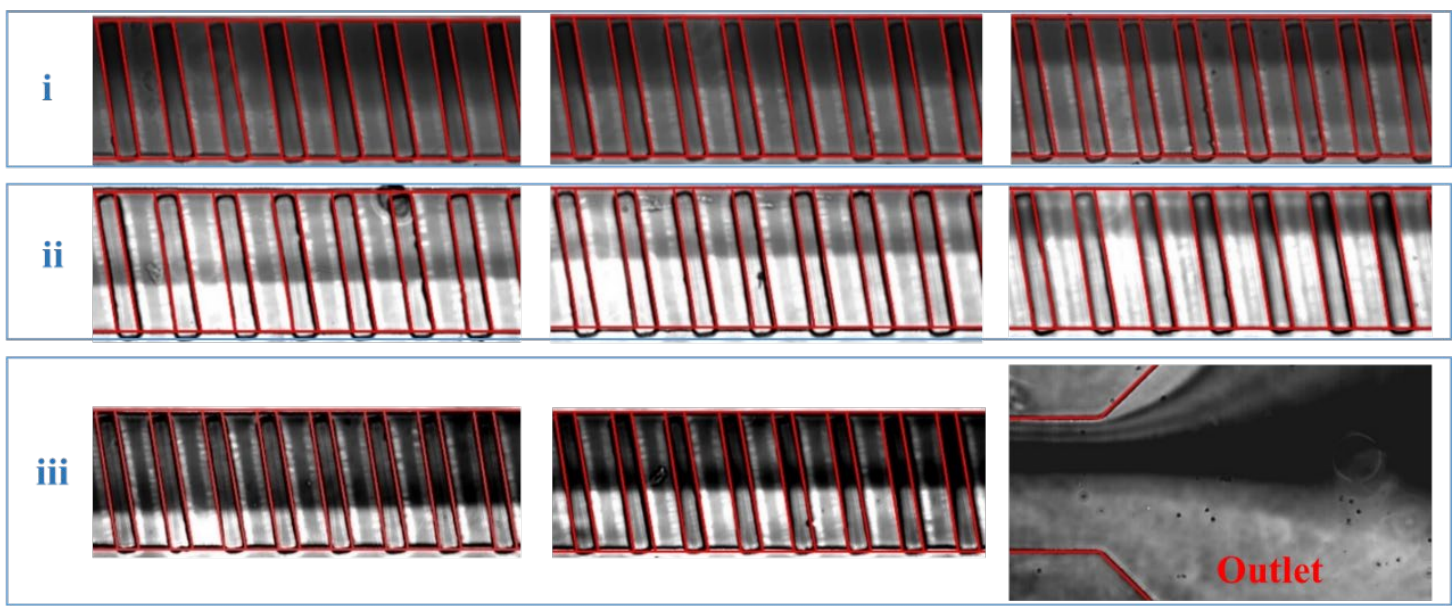

(b)

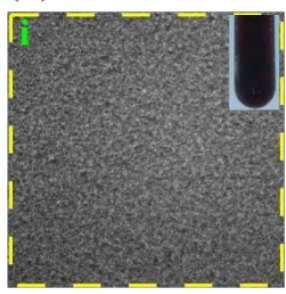

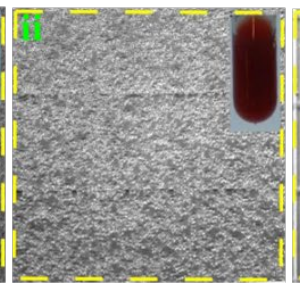

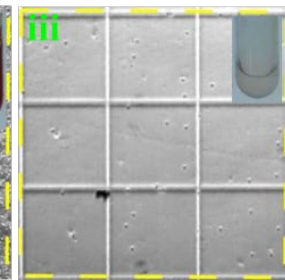

(c)

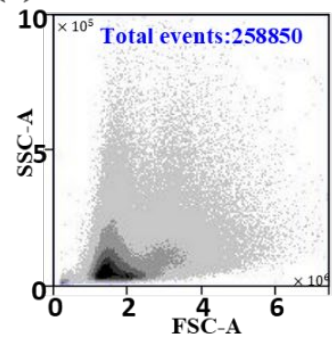

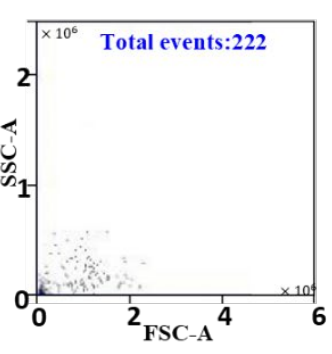

Fig. 4 Human blood plasma extraction from the whole blood (a)Plasma extraction from the whole blood (45\% hct) at the total flow rate of $500 \mu \mathrm{l} / \mathrm{min}$ and the flow rate ratio of 4 (i); Plasma extraction from the diluted blood $(5.63 \% \mathrm{hct})$ at the total flow rate of $500 \mu \mathrm{l} / \mathrm{min}$ and the flow rate ratio of 4 (ii); Plasma extraction from the whole blood ( $45 \% \mathrm{hct}$ ) at the total flow rate of $700 \mu \mathrm{l} / \mathrm{min}$ and the flow rate ratio of 6 (iii). (b)The bright field images of (i) the whole blood sample before extraction, (ii) the extracted blood cells solution and (iii) extracted blood plasma on the haemocytometer. (c)The achieved data from the flow cytometry were processed and analysed by the software of FlowJo (Tree Star, Inc.). The X axis (SSC-A) represented the relative cell surface and intracellular complexity and the $\mathrm{Y}$-axis (FSC-A) represented the relative event size. 
Table. 1 Comparison of typical microfluidic techniques for blood plasma extraction.

\begin{tabular}{|c|c|c|c|}
\hline Method & Sample Throughput & Dilution & Purity \\
\hline Inertial (spiral) $[24,25]$ & $700 \mu \mathrm{l} / \mathrm{min}$ & $5 \%$ & $100 \%$ \\
\hline Inertial (serpentine)[26] & $350 \mu \mathrm{l} / \mathrm{min}$ & $5 \%$ & $99.75 \%$ \\
\hline Inertial (this work) & $700 \mu \mathrm{l} / \mathrm{min}$ & $100 \%$ & $99.9 \%$ \\
\hline Hydrodynamic (DLD)[21] & $0.4 \mu \mathrm{l} / \mathrm{min}$ & $100 \%$ & $100 \%$ \\
\hline Hydrodynamic (Centrifugal)[27] & $15 \mu \mathrm{l} / \mathrm{min}$ & $100 \%$ & $98.9 \%$ \\
\hline Dielectrophoresis[18] & $0.5 \mu \mathrm{l} / \mathrm{min}$ & $11.1 \%$ & $97 \%$ \\
\hline Acoustophoresis[28] & $80 \mu \mathrm{l} / \mathrm{min}$ & $20 \%$ & $98 \%$ \\
\hline
\end{tabular}

